\title{
A Direct Eulerian GRP Scheme for the Prediction of Gas-Liquid Two-Phase Flow in HTHP Transient Wells
}

\author{
Jiuping Xu, ${ }^{1}$ Min Luo, ${ }^{1,2}$ Jiancheng Hu, ${ }^{1,2}$ Shize Wang, ${ }^{3}$ Bin Qi, $^{3}$ and Zhiguo Qiao ${ }^{3}$ \\ ${ }^{1}$ Uncertainty Decision-Making Laboratory, Sichuan University, Chengdu 610064, China \\ ${ }^{2}$ College of Mathematics, Chengdu University of Information Technology, Chengdu 610225, China \\ ${ }^{3}$ Research School of Engineering Technology, China Petroleum and Chemical Corporation, Deyang 618000, China \\ Correspondence should be addressed to Jiuping Xu; xujiuping@scu.edu.cn
}

Received 10 September 2013; Accepted 17 October 2013

Academic Editor: Mohamed Fathy El-Amin

Copyright (C) 2013 Jiuping Xu et al. This is an open access article distributed under the Creative Commons Attribution License, which permits unrestricted use, distribution, and reproduction in any medium, provided the original work is properly cited.

A coupled system model of partial differential equations is presented in this paper, which concerns the variation of the pressure and temperature, velocity, and density at different times and depths in high temperature-high pressure (HTHP) gas-liquid two-phase flow wells. A new dimensional splitting technique with Eulerian generalized riemann problem (GRP) scheme is applied to solve this set of conservation equations, where Riemann invariants are introduced as the main ingredient to resolve the generalized Riemann problem. The basic data of "X well" (HTHP well), $7100 \mathrm{~m}$ deep, located in Southwest China, is used for the case history calculations. Curve graphs of pressures and temperatures along the depth of the well are plotted at different times. The comparison with the results of Lax Friedrichs $(\mathrm{LxF})$ method shows that the calculating results are more fitting to the values of real measurement and the new method is of high accuracy.

\section{Introduction}

The prediction of pressure and temperature of transient gasliquid flow in a wellbore is important but difficult for well completion test because they are characterized by the dependence of pressure, density, velocity, and other flow parameters on both time and space. As for pressure prediction research, there exist empirical formulas, such as those given by Beggs and Brill [1], Mukherjee and Brill [2, 3], and so on. Different researchers such as Hurlburt and Hanratty [4] and CazarezCandia and Vásquez-Cruz [5] have proposed mechanistic models, assuming that flow is under steady-state conditions; other researchers such as Taitel et al. [6]; Ouyang and Aziz [7] have proposed unsteady-state gas-liquid two-phase flow models. Fontanilla and Aziz [8] and Ali [9] presented two simultaneous ordinary differential equations for estimating the steam pressure and quality and solved these equations by using the fourth-order Runge-Kutta method. However, those models can only predict the pressure profiles but not the temperature profiles and ignored their interdependence.

Concerning both pressure and temperature in HTHP wells, $\mathrm{Wu}$ et al. have presented a coupled system model of differential equations in [10], but this model only considered the single phase flow statement. In this paper, we build a set of coupled partial differential equations of pressure, temperature, density, and velocity in HTHP gas-liquid twophase flow wells on the base of the model which was build by $\mathrm{Xu}$ et al. in [11]. The numerical model, which accords with the actual situation of the well, allows for the change of oblique angle,different heat transfer medium in annular and the depth of the physical properties of the formation.

We found an algorithm solving model with generalized Riemann problem (GRP) scheme, which is an analytic extension of the Godunov scheme in [12] and originally designed by $\mathrm{Li}$ and Chen in [13] for the shallow water equations. A direct and simple derivation of the Eulerian generalized Riemann problem scheme is presented to get the integration in time of the conservation laws. Riemann invariants are applied in order to resolve the singularity at the jump discontinuity. The approach has the advantage that the contact discontinuity in each local wave pattern is always fixed with speed zero, while the rarefaction and the shock waves are located on either side. Since the extension of this scheme to multidimensional cases is obtained using 


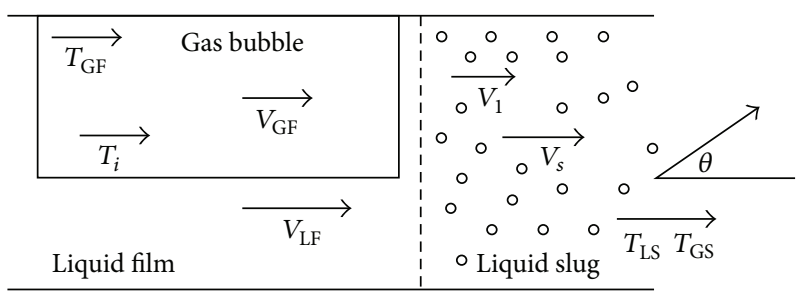

FIGURE 1: The two-phase flow.

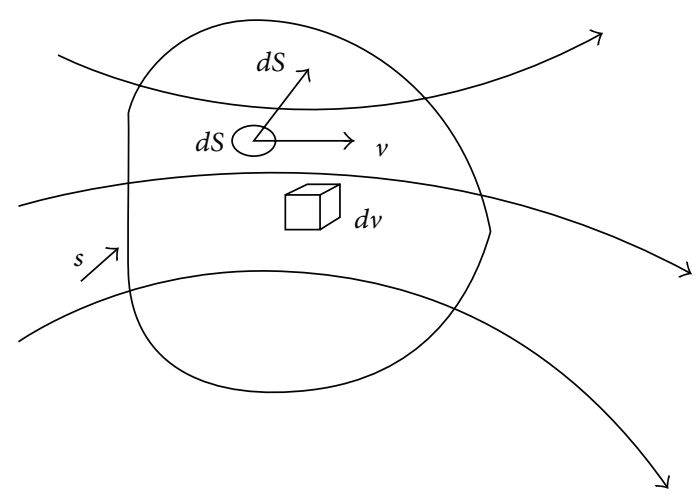

Figure 2: Control volume 1.

the dimensional splitting technique, getting the integration in time of the conservation laws is more direct and simple.

In this paper, we use GRP method for solving this problem and get more accurate prediction of pressure and temperature compared with those obtained from the existing correlations such as LxF method in [11]. The basic data for the calculation are from X well, $7100 \mathrm{~m}$ of depth in Southwest China. The curves of the gas pressure and temperature along the depth of the well are plotted. The results can provide a technical reliance for the process of designing well tests in HTHP gas-liquid wells and a dynamic analysis of production from wells.

\section{Model Formulation}

Considering the two-phase flow system shown in Figure 1, the mixture density and velocity are related to the in situ liquid volume fraction (holdup), $H$, as follows:

$$
\begin{gathered}
\rho_{m}=\rho_{l} H+\rho_{g}(1-H) \\
v_{m}=v_{l} H+v_{g}(1-H) \\
u_{m}=u_{l} H+u_{g}(1-H) .
\end{gathered}
$$

2.1. Mass Balance. Consider the flow model shown in Figure 2. According to the fluid moves through the fixed control volume depicted by John and Anderson in [14], we have

$$
\iint_{S} \rho_{m} v_{m} d s=-\frac{\partial}{\partial t} \iiint_{v} \rho_{m} d v_{m} .
$$

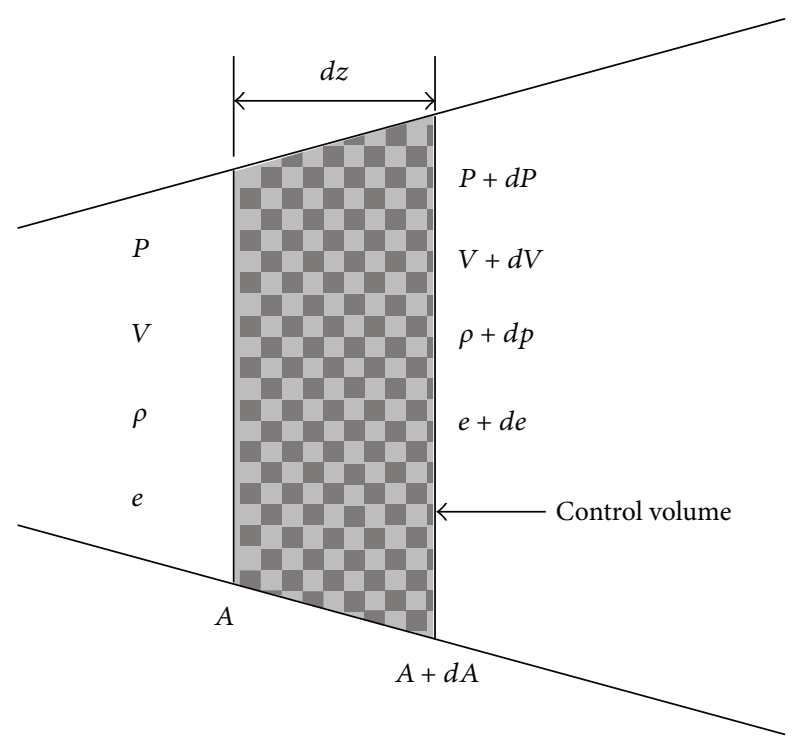

FIgURE 3: Control volume 2.

Under transient conditions, applied to the control volume in Figure 3, in the limit as $d z$ becomes very small, the volume and surface integral in (2) becomes

$$
\begin{gathered}
\frac{\partial}{\partial t} \iiint_{v} \rho_{m} d v=\frac{\partial}{\partial t}\left(\rho_{m} A d z\right) \\
\iint_{S} \rho_{m} v_{m} d s=\rho_{m} v_{m} d v_{m}+\rho_{m} A d v_{m}+A v_{m} d \rho_{m} \\
=d\left(\rho_{m} v_{m} A\right) .
\end{gathered}
$$

Substituting (3) into (2), we get the mass balance equation:

$$
\frac{\partial \rho_{m}}{\partial t}+\frac{\partial\left(\rho_{m} v_{m}\right)}{\partial z}=0
$$

2.2. Momentum Balance. As shown in Figure 4, the integral form of the $z$ component the momentum equation can be written as follows with the external forces:

$$
\begin{aligned}
\frac{\partial}{\partial t} & \iiint_{v} \rho_{m} u_{m} d V+\iint_{S}\left(\rho_{m} u_{m} v_{m}\right) \cdot d S \\
& =-\iint_{S}(P d S) d z-\rho_{m} g \cos \theta A d z-\frac{\lambda \rho_{m} v_{m}^{2}}{2 d} A d z,
\end{aligned}
$$

where $\rho_{m} g \cos \theta A d z$ is the force of gravity, $\left(\lambda \rho_{m} v^{2} A / 2\right) d z$ is the shear stress, and

$$
\frac{\partial}{\partial t} \iiint_{v} \rho_{m} u_{m} d V=\frac{\partial}{\partial t}\left(\rho_{m} v_{m} A d z\right)
$$

$$
\begin{aligned}
\iint_{S}\left(\rho_{m} u_{m} v_{m}\right) \cdot d S= & -\rho_{m} v_{m}^{2} A \\
& +\left(\rho_{m}+d \rho_{m}\right)\left(v_{m}+d v_{m}\right)^{2}(A+d A) \\
-\iint_{S}(P d S)_{z}=-P A & +(P+d P)(A+d A)-2 P\left(\frac{d A}{2}\right) .
\end{aligned}
$$




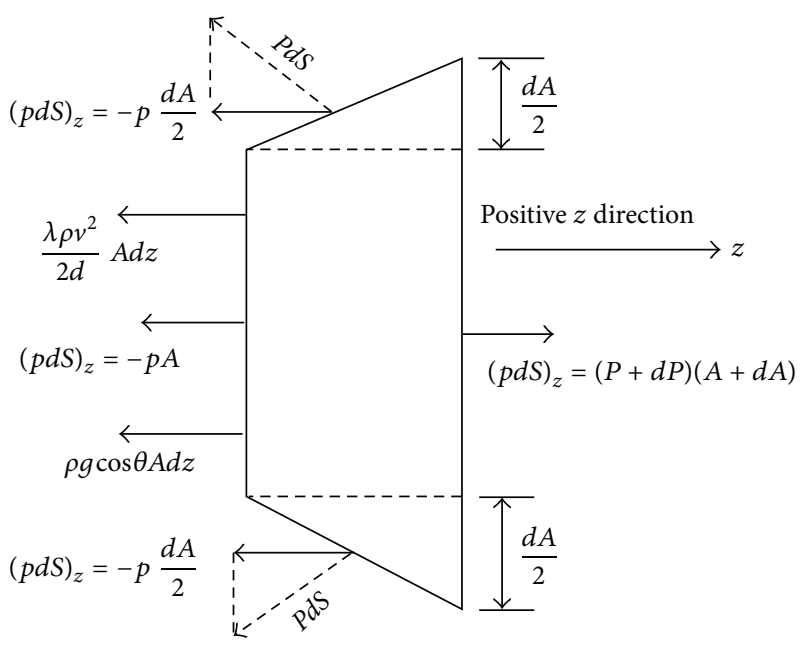

FIGURE 4: Control volume 3.

Substituting (6) into (5), we obtain momentum balance equation:

$$
\frac{\partial}{\partial t}\left(\rho_{m} v_{m}\right)+\frac{\partial}{\partial z}\left(P+\rho_{m} v_{m}^{2}\right)=-\rho_{m} g \cos \theta-\frac{\lambda \rho_{m} v_{m}^{2}}{2 d}
$$

2.3. Energy Balance. For the transient flow, it leads directly to the energy equation in terms of temperature. As shown in Figure 5, we should consider the heat transmission within wellbore and from wellbore to formation as transient.

According to the energy balance law, the heat variation flowing on control volume that is equal to the combination heat of inflow and outflow, and the heat transferring to the second dimension, we get the energy balance equation of transient flow:

$$
\begin{gathered}
\left(w C_{p} T\right) z-\left(w C_{p} T\right)(z+d z)-2 \pi r_{t o} U_{t o}\left(T-T_{r}\right) d z \\
=\frac{\partial\left(\rho_{m} C_{p} T\right)}{\partial t} A d z
\end{gathered}
$$

where $T_{r}=\left(K_{e} T_{e i}+r_{t o} U_{t o} T_{e} T_{\mathrm{wbD}}\right) /\left(K_{e}+r_{t o} U_{t o} T_{\mathrm{wbD}}\right)$ and $w=\rho_{m} v_{m} A$. Equation (8) equals the following equation:

$$
\frac{\partial\left(\rho_{m} v_{m} T\right)}{\partial z}+\frac{\partial\left(\rho_{m} T\right)}{\partial t}=\frac{2 \pi K_{e} r_{t o} U_{t o}\left(T_{e i}-T\right)}{C_{P}\left(K_{e}+r_{t o} U_{t o} T_{\mathrm{wbD}}\right)}
$$

Finally, we obtain the coupled system model of partial differential equations:

$$
\begin{gathered}
\frac{\partial \rho_{m}}{\partial t}+\frac{\partial\left(\rho_{m} v_{m}\right)}{\partial z}=0 \\
\frac{\partial\left(\rho_{m} v_{m}\right)}{\partial t}+\frac{\partial\left(P+\rho_{m} v_{m}^{2}\right)}{\partial z}=-\rho_{m} g \cos \theta-\frac{\lambda}{2 d}
\end{gathered}
$$

$$
\begin{gathered}
\frac{\partial\left(\rho_{m} T\right)}{\partial t}+\frac{\partial\left(\rho_{m} v_{m} T\right)}{\partial z}=\frac{2 \pi K_{e} r_{t o} U_{t o}\left(T_{e i}-T\right)}{C_{P}\left(K_{e}+r_{t o} U_{t o} T_{\mathrm{wbD}}\right)}, \\
\rho_{m}=\frac{M P \gamma_{g}}{Z R T}, \\
\left(P, T, \rho_{m}, v_{m}\right)=\left(P_{0}, T_{0}, \rho_{m 0}, v_{m 0}\right) \quad t=0, z=0 .
\end{gathered}
$$

\section{Format Construction}

We unify the conservation equations (4), (7), and (9) which are also included in (10) into the following formation:

$$
\begin{gathered}
\quad \frac{\partial A_{m}}{\partial t}+\frac{\partial B_{m}}{\partial z}=C_{m}, \quad m=1,2,3, \\
U=\left\{\begin{array}{l}
A_{1}=\rho_{m} \\
A_{2}=\rho_{m} v_{m} \\
A_{3}=0,
\end{array} \quad F(U)=\left\{\begin{array}{l}
B_{1}=\rho_{m} v_{m} \\
B_{2}=P+\rho_{m} v_{m}^{2} \\
B_{3}=\rho_{m} v_{m} T,
\end{array}\right.\right. \\
S(U)=\left\{\begin{array}{l}
C_{1}=0 \\
C_{2}=-\rho_{m} g \cos \theta-\frac{\lambda}{2 d} \\
C_{3}=\frac{2 \pi K_{e} r_{t o} U_{t o}\left(T_{e i}-T\right)}{C_{P}\left(K_{e}+r_{t o} U_{t o} T_{\mathrm{wbD}}\right)} .
\end{array}\right.
\end{gathered}
$$

We define the equally spaced grid points, the interface points, and the cells as

$$
z_{j}=j \Delta z, \quad z_{j+1 / 2}=\frac{z_{j}+z_{j+1}}{2}, \quad C_{j}=\left[z_{j-1 / 2}, z_{j+1 / 2}\right] .
$$

We assume that the data at time $t=t_{n}$ are piecewise linear with a slope $\sigma_{j}^{n}$ and we have $U\left(z, t_{n}\right)=U_{j}^{n}+\sigma_{j}^{n}(z-$ $\left.z_{j}\right), z \in\left(z_{j-1 / 2}, z_{j+1 / 2}\right)$.

The second-order Godunov scheme for (11) takes the following form: $U_{j}^{n+1}=U_{j}^{n}-(\Delta t / \Delta x)\left(F\left(U_{j+1 / 2}^{n+1 / 2}\right)-F\left(U_{j-1 / 2}^{n+1 / 2}\right)\right)$, where $U_{j+1 / 2}^{n+1 / 2}$ is the midpoint value or the value of $U$ at the cell interface $\left(z_{j+1 / 2}, t_{n}\right)$ with accuracy of second order. More specifically, the mid-point value $U_{j+1 / 2}^{n+1 / 2}$ is computed with the formulas $U_{j+1 / 2}^{n+1}=U_{j+1 / 2}^{n}+(\Delta t / 2)(\partial U / \partial t)_{j+1 / 2}^{n}$ and $U_{j+1 / 2}^{n}=R^{A}\left(0 ; U_{j+1 / 2,-}^{n}, U_{j+1 / 2,+}^{n}\right)$. Also, $R^{A}\left(\left(z-z_{j+1 / 2}\right) /(t-\right.$ $\left.\left.t_{n}\right) ; U_{j+1 / 2,-}^{n}, U_{j+1 / 2,+}^{n}\right)$ is the solution of the Riemann problem centered at $\left(z_{j+1 / 2}, t_{n}\right)$. Moreover, $U_{j+1 / 2,-}^{n}$ and $U_{j+1 / 2,+}^{n}$ are the limiting values of initial data $U\left(z, t_{n}\right)$ on both sides of $\left(z_{j+1 / 2}, t_{n}\right)$. We present a direct and simple derivation of the Eulerian geralized Riemann problem (GRP) scheme and apply Riemann invariants in order to resolve the singularity at the jump discontinuity.

The local wave configuration is usually piecewise smooth and consists of rarefaction waves, shocks, and contact discontinuities. As the general rarefaction waves are considered, the initial data can be regarded as a perturbation of the Riemann initial data $U_{L}$ and $U_{R}$. 


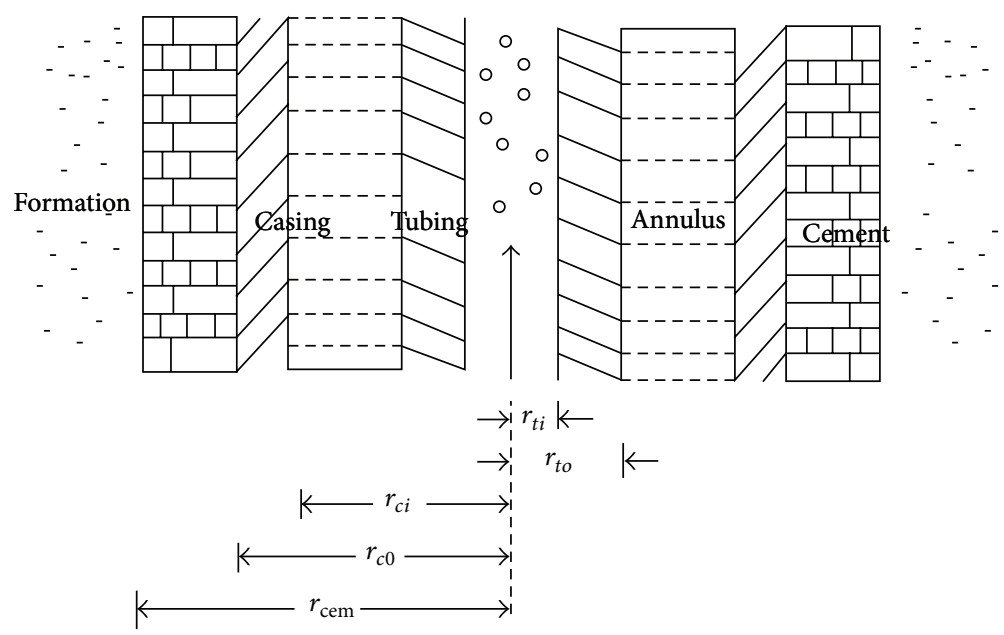

Figure 5: The radial transfer of heat.

The GRP scheme assumes piecewise linear data for the flow variables, which leads to the generalized Riemann problem for (11) subject to the initial data:

$$
U(z, 0)= \begin{cases}U_{L}+z U_{L}^{\prime}, & z<0 \\ U_{R}+z U_{R}^{\prime}, & z>0,\end{cases}
$$

where $U_{L}, U_{R}, U_{L}^{\prime}$, and $U_{R}^{\prime}$ are constant vectors.

The initial structure of the solution is determined by the associated Riemann solution, denoted by $\lim _{t \rightarrow 0} U(\lambda t, t)=$ $R^{A}\left(\lambda ; U_{L}, U_{R}\right), \lambda=x / t$.

\section{Solving Process}

Step 1. Set the step length. In this paper,

$$
h=1(\mathrm{~m}), \quad \tau=60(\mathrm{~s}) .
$$

Step 2. Obtain each point's inclination: $\theta_{j}=\theta_{j-1}+\left(\theta_{j^{\prime}}-\right.$ $\left.\theta_{j^{\prime}-1}\right) h / \Delta s_{j^{\prime}-1}$.

Step 3. The in situ liquid volume fraction (holdup) in (1) can be calculated from

$$
\begin{aligned}
H\left(\theta_{j}\right)= & \frac{0.98 E_{l}^{0.4846}}{F r^{0.0868}} \\
& \times\left\{1+\left(1-E_{l}\right) \ln \left[\frac{4.7 N_{v l}^{0.1244}}{E_{l}^{0.8692} \mathrm{Fr}^{0.5056}}\right]\right. \\
& \left.\times\left[\sin \left(1.8 \theta_{j}\right)-\frac{1}{3} \sin ^{3}\left(1.8 \theta_{j}\right)\right]\right\} .
\end{aligned}
$$

Step 4. Calculate the following parameters by Liao and Feng in $[15]$ :

$$
\begin{gathered}
U_{t o}=\left(\frac{r_{t o}}{r_{t i} h_{r}}+\frac{r_{t o} \ln \left(r_{t o} / r_{t i}\right)}{r_{t i} h_{r}}+\frac{1}{h_{c}+h_{r}}\right. \\
\left.+\frac{r_{t o} \ln \left(r_{c 0} / r_{c i}\right)}{r_{\mathrm{cas}}}+\frac{r_{t o} \ln \left(r_{h} / r_{c 0}\right)}{r_{\mathrm{cem}}}\right)^{-1},
\end{gathered}
$$

$$
T_{\mathrm{wbD}}=\frac{2 \pi K_{e}\left(T_{e i}-T_{\mathrm{wb}}\right)}{\sum_{j=1}^{m}\left(Q_{j-1}-Q_{j}\right)} .
$$

Step 5. For piecewise given initial data $U^{n}(z)=U_{j}^{n}+\sigma_{j}^{n}(z-$ $\left.z_{j}\right), z \in\left(z_{j-1 / 2}, z_{j+1 / 2}\right)$, we solve the Riemann problem for (11) to define the Riemann solution $U_{j+1 / 2}^{n}=R\left(0 ; U_{j}^{n}+\right.$ $\left.(\Delta z / 2) \sigma_{j}^{n}, U_{j+1}^{n}-(\Delta z / 2) \sigma_{j+1}^{n}\right)$, which is the same as the classical Godunov scheme and the Riemann solver in [16] is used in the solution.

Step 6. Determine $(\partial U / \partial t)_{j+1 / 2}^{n}$ and evaluate the new cell averages $U_{j}^{n+1}$. We apply monotonic algorithm slope limiters to suppress the local oscillations near discontinuities. We use parameter $\alpha=1.9$ in $\sigma_{j}^{n+1}=\min \bmod \left(\alpha\left(U_{j}^{n+1}-\right.\right.$ $\left.\left.U_{j-1}^{n+1}\right) / \Delta z, \sigma_{j}^{n+1,-}, \alpha\left(U_{j+1}^{n+1}-U_{j}^{n+1}\right) / \Delta z\right)$, where $U_{j+1 / 2}^{n+1,-}=$ $U_{j+1 / 2}^{n}+\Delta z(\partial U / \partial z)_{j+1 / 2}^{n}$ and $\sigma_{j}^{n+1,-}=(1 / \Delta z)(\Delta U)_{j}^{n+1,-}=$ $(1 / \Delta z)\left(U_{j+1 / 2}^{n+1,-}-U_{j-1 / 2}^{n+1,-}\right)$.

\section{Results and Discussion}

In this simulation, we study a pipe in X well located in Sichuan Basin, Southwest China. All the needed parameters are given in [17] as follows: fluid density is $1000 \mathrm{~kg} / \mathrm{m}^{3}$; depth of the well is $7100 \mathrm{~m}$; friction coefficient is 1.2 ; ground temperature is $160^{\circ} \mathrm{C}$; ground thermal conductivity parameter is 2.06 ; ground temperature gradient is $0.0218^{\circ} \mathrm{C} / \mathrm{m}$. Parameters of pipes are given in Table 1 . Inclination, azimuth, and vertical depth are given in Table 2.

Through the simulation, we use GRP method to calculate the prediction of pressure and temperature of the oil in the pipe and draw a sensitive analysis for the results. We compare the results of pressure and temperature calculated for the well head at 1200 s by GRP and LxF scheme with the measurement results, which also shows that GRP scheme is more accurate 
TABLE 1: Parameters of pipes.

\begin{tabular}{lcccccc}
\hline Diameter & Thickness & Weight & Expansion & Coefficient & Young & Modulus \\
\hline 88.9 & 9.53 & 18.9 & 0.0000115 & 215 & 215 & 0.3 \\
88.9 & 7.34 & 15.18 & 0.0000115 & 215 & 0.3 & 750 \\
88.9 & 6.45 & 13.69 & 0.0000115 & 215 & 0.3 & 4200 \\
73 & 7.82 & 12.8 & 0.0000115 & 215 & 0.3 & 0.3 \\
73 & 5.51 & 9.52 & 0.0000115 & & 150 \\
\hline
\end{tabular}

TABle 2: Parameters of azimuth, inclination, and vertical depth.

\begin{tabular}{|c|c|c|c|c|}
\hline Number & Measured & Inclination & Azimuth & $\begin{array}{c}\text { Vertical } \\
\text { depth }\end{array}$ \\
\hline 1 & 0 & 0 & 120.33 & 0 \\
\hline 2 & 303 & 1.97 & 121.2 & 302.87 \\
\hline 3 & 600 & 1.93 & 120.28 & 599.73 \\
\hline 4 & 899 & 0.75 & 126.57 & 898.59 \\
\hline 5 & 1206 & 1.25 & 124.9 & 1205.45 \\
\hline 6 & 1505 & 1.04 & 124.62 & 1504.32 \\
\hline 7 & 1800 & 0.49 & 123.75 & 1799.18 \\
\hline 8 & 2105 & 2.49 & 125.27 & 2104.04 \\
\hline 9 & 2401 & 1.27 & 123.13 & 2399.91 \\
\hline 10 & 2669 & 2.44 & 120.12 & 2667.79 \\
\hline 11 & 3021 & 0.14 & 127.39 & 3019.63 \\
\hline 12 & 3299 & 1.18 & 122.60 & 3297.50 \\
\hline 13 & 3605 & 2.05 & 123.25 & 3603.36 \\
\hline 14 & 3901 & 0.16 & 121.45 & 3899.22 \\
\hline 15 & 4183 & 2.92 & 121.24 & 4181.09 \\
\hline 16 & 4492 & 2.73 & 129.22 & 4489.95 \\
\hline 17 & 4816.07 & 1.98 & 121.61 & 4813.87 \\
\hline 18 & 5099.07 & 2.74 & 129.93 & 5096.74 \\
\hline 19 & 5394.07 & 0.13 & 120.46 & 5391.61 \\
\hline 20 & 5706.07 & 0.63 & 129.59 & 5703.47 \\
\hline 21 & 5983.07 & 2.09 & 120.14 & 5980.34 \\
\hline 22 & 6302.07 & 2.69 & 122.91 & 6299.19 \\
\hline 23 & 6597.07 & 2.45 & 129.41 & 6594.06 \\
\hline 24 & 6911.12 & 0.15 & 124.88 & 6907.96 \\
\hline
\end{tabular}

in the real calculation. We obtain series of results contained in tables and figures and analyze these results as follows.

When the bottom pressure is $70 \mathrm{MPa}$, temperatures are plotted in Figure 6 at different depths and shown in detail in Table 3. When the output keeps constant, the temperature increases with the increasing depth of the well and when the depth fixed, the temperature increases with the increasing time. In addition, it can be seen from the figure that the temperature changes quickly in the early stage but stabilizes over time, especially after $1200 \mathrm{~s}$.

It is established that, when depth is constant, the pressure shown in Figure 7 and Table 4 increased with an increase of the time. When the output keeps constant, the pressure increased with the increasing depth of the well. This is because, with time increasing, the flow increases and then the

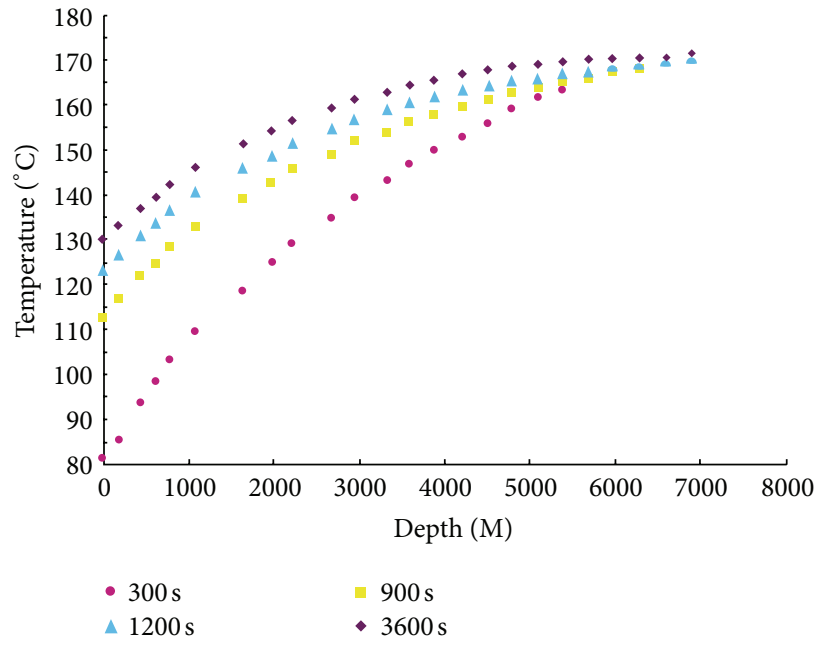

FIGURE 6: Temperature distribution at different depths.

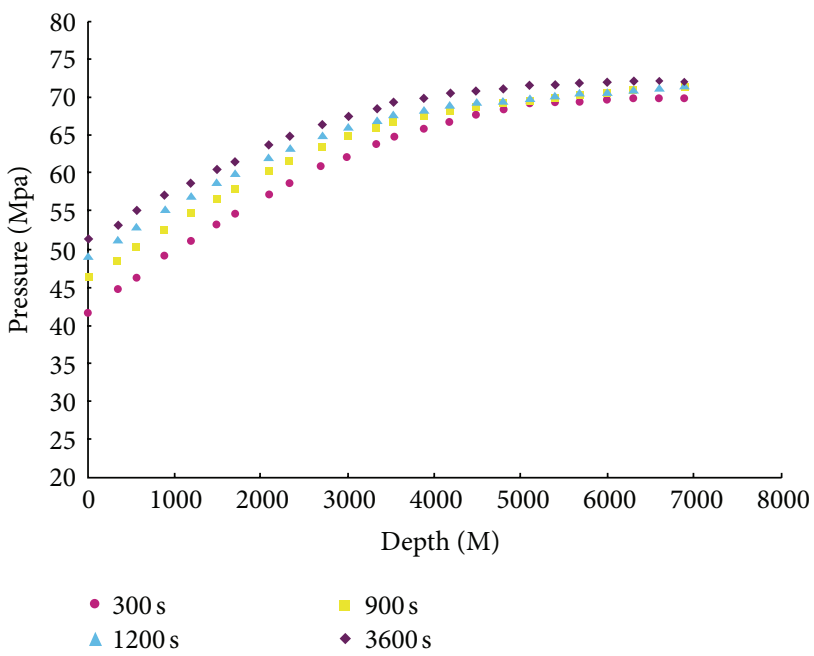

FIGURE 7: Pressure distribution at different depths.

frictional heat leads to an increase in the pressure. It can also be seen that the pressure changes quickly in the early stage but stabilizes over time.

As shown in Table 5, for the comparative results of the well head temperature at $1200 \mathrm{~s}$, the relative error between the calculation results and the measurement results of GRP scheme method is $5.12 \%$ and by LxF method is $6.70 \%$, while the relative error between the results in pressure predition at 
TABLE 3: Temperature at different depths on $300 \mathrm{~s}, 900 \mathrm{~s}, 1200 \mathrm{~s}$, and $3600 \mathrm{~s}$.

\begin{tabular}{|c|c|c|c|c|}
\hline \multirow{2}{*}{ Depth } & \multicolumn{4}{|c|}{ Time } \\
\hline & $300 \mathrm{~s}$ & $900 \mathrm{~s}$ & $1200 \mathrm{~s}$ & $3600 \mathrm{~s}$ \\
\hline 0 & 81.22 & 115.29 & 124.19 & 132.27 \\
\hline 300 & 85.45 & 121.38 & 127.55 & 133.34 \\
\hline 600 & 92.67 & 125.54 & 131.76 & 136.56 \\
\hline 900 & 95.54 & 129.48 & 134.96 & 138.87 \\
\hline 1200 & 101.16 & 133.77 & 137.58 & 141.94 \\
\hline 1500 & 106.49 & 136.66 & 140.77 & 143.15 \\
\hline 1800 & 111.76 & 140.67 & 143.23 & 145.17 \\
\hline 2100 & 116.98 & 143.54 & 146.29 & 147.39 \\
\hline 2400 & 121.86 & 145.66 & 148.49 & 149.14 \\
\hline 2700 & 126.89 & 148.45 & 150.78 & 151.93 \\
\hline 3000 & 131.55 & 151.78 & 152.99 & 153.85 \\
\hline 3300 & 136.85 & 153.74 & 154.86 & 155.91 \\
\hline 3600 & 140.88 & 154.02 & 154.46 & 157.87 \\
\hline 3900 & 144.67 & 157.12 & 158.73 & 159.45 \\
\hline 4200 & 148.25 & 159.34 & 160.87 & 161.88 \\
\hline 4500 & 152.74 & 161.53 & 161.65 & 162.65 \\
\hline 4800 & 155.77 & 162.55 & 162.72 & 163.45 \\
\hline 5100 & 159.75 & 163.42 & 163.49 & 164.56 \\
\hline 5400 & 162.36 & 164.56 & 164.87 & 165.24 \\
\hline 5700 & 164.32 & 165.74 & 165.45 & 166.57 \\
\hline 6000 & 166.36 & 166.56 & 167.67 & 167.97 \\
\hline 6300 & 167.91 & 168.77 & 168.87 & 169.65 \\
\hline 6600 & 168.23 & 169.45 & 169.57 & 169.81 \\
\hline 6900 & 170.24 & 170.56 & 171.78 & 171.52 \\
\hline
\end{tabular}

the same time calculated by GRP scheme method is $8.81 \%$ and by $\mathrm{LxF}$ method is $9.73 \%$, which shows that the distribution prediction of the two-phase flow is more accurate in actual calculation by GRP scheme method.

\section{Conclusion}

In this paper, considering the variation of pressure, temperature, velocity; and density at different times and depths in gasliquid two-phase flow, we present a system model of partial differential equations according to mass, momentum, and energy. We establish an algorithm solving model with a new difference method with a direct Eulerian GRP scheme which is proven to be efficient for the numerical implementation in this paper. The basic data of the X well (HTHP well), $7100 \mathrm{~m}$ deep in Sichuan Basin, Southwest China, was used for case history calculations, and a sensitivity analysis is completed for the model. The gas-liquid's pressure and temperature curves along the depth of the well are plotted, and the curves intuitively reflect the flow law and the characteristics of heat transfer in formation. The results can provide the technical reliance for the process of designing well tests in high temperature-high pressure gas-liquid two-phase flow wells and dynamic analysis of production. Furthermore, the works in this paper can raise safety and reliability of deep completion test and will yield notable economic and social
TABle 4: Pressure at different depths on 300 s, 900 s, 1200 s, and $3600 \mathrm{~s}$.

\begin{tabular}{|c|c|c|c|c|}
\hline \multirow{2}{*}{ Depth } & \multicolumn{4}{|c|}{ Time } \\
\hline & $300 \mathrm{~s}$ & $900 \mathrm{~s}$ & $1200 \mathrm{~s}$ & $3600 \mathrm{~s}$ \\
\hline 0 & 42.55 & 46.62 & 50.24 & 51.34 \\
\hline 300 & 43.23 & 47.53 & 50.64 & 52.67 \\
\hline 600 & 44.86 & 48.71 & 50.46 & 53.47 \\
\hline 900 & 45.87 & 49.13 & 51.41 & 54.69 \\
\hline 1200 & 46.73 & 50.43 & 52.32 & 54.79 \\
\hline 1500 & 48.46 & 51.24 & 53.36 & 55.53 \\
\hline 1800 & 49.43 & 52.43 & 54.47 & 56.12 \\
\hline 2100 & 50.34 & 53.83 & 55.42 & 57.37 \\
\hline 2400 & 51.96 & 56.92 & 54.37 & 57.85 \\
\hline 2700 & 53.53 & 57.22 & 55.45 & 58.97 \\
\hline 3000 & 54.44 & 58.46 & 56.78 & 59.34 \\
\hline 3300 & 55.24 & 59.97 & 57.47 & 60.95 \\
\hline 3600 & 56.76 & 59.94 & 58.95 & 61.22 \\
\hline 3900 & 57.33 & 60.98 & 59.04 & 62.29 \\
\hline 4200 & 58.93 & 61.22 & 60.29 & 63.33 \\
\hline 4500 & 59.34 & 62.45 & 61.24 & 64.48 \\
\hline 4800 & 60.89 & 63.43 & 62.23 & 64.33 \\
\hline 5100 & 61.56 & 64.19 & 63.22 & 64.78 \\
\hline 5400 & 63.35 & 65.24 & 64.18 & 65.34 \\
\hline 5700 & 64.69 & 65.45 & 65.12 & 66.34 \\
\hline 6000 & 65.45 & 66.79 & 66.15 & 67.56 \\
\hline 6300 & 66.99 & 67.49 & 67.11 & 67.47 \\
\hline 6600 & 67.46 & 68.52 & 68.22 & 68.58 \\
\hline 6900 & 69.28 & 69.46 & 69.92 & 69.55 \\
\hline
\end{tabular}

TABLE 5: Comparative results of the well head at $1200 \mathrm{~s}$.

\begin{tabular}{lcc}
\hline Well-head & Temperature & Pressure \\
\hline $\begin{array}{l}\text { Measurement results } \\
\begin{array}{l}\text { Results by GRP method } \\
\text { (relative error) }\end{array}\end{array}$ & 180.65 & 76.10 \\
$\begin{array}{l}\text { Results by LxF method } \\
\text { (relative error) }\end{array}$ & $169.30(6.70 \%)$ & $69.36(9.73 \%)$ \\
\hline
\end{tabular}

benefits and avoid or lessen accidents caused by improper technical design.

\section{Nomenclature}

A: A total length of conduit $\left(\mathrm{m}^{2}\right)$

$C_{J}$ : Joule-Thompson coefficient $(\mathrm{K} / \mathrm{pa})$

$C_{p}$ : Heat capacity $(\mathrm{J} / \mathrm{kg} \cdot \mathrm{K})$

$D$ : A hydraulic diameter $(\mathrm{m})$

G: Acceleration constant of gravity $\left(\mathrm{m} / \mathrm{s}^{2}\right)$

$K_{e}:$ Formation conductivity $(\mathrm{J} / \mathrm{m} \cdot \mathrm{K})$

$P$ : Pressure (KPa)

$r_{D}$ : Dimensionless radius

$r_{t o}:$ Outer radius of conduit $(\mathrm{m})$

$T: \quad$ Temperature $(\mathrm{K})$

$t_{D}$ : Dimensionless time 
$T_{e}: \quad$ Temperature of the stratum $(\mathrm{K})$

$T_{\mathrm{wb}}$ : Wellbore temperature (K)

$T_{\mathrm{wbD}}$ : Dimensionless wellbore temperature (K)

$T_{r}: \quad$ Temperature of the second surface $(\mathrm{K})$

$T_{e i}: \quad$ Initial temperature of formation (K)

$U_{t o}: \quad$ Overall-heat-transfer coefficient $(\mathrm{W} / \mathrm{m} \cdot \mathrm{K})$

$V: \quad$ Velocity $(\mathrm{m} / \mathrm{s})$

$Z: \quad$ A total length of conduit (m)

$z: \quad$ The distance coordinate in the direction along the conduit

$h_{c}$ : Heat transfer coefficient for natural convection based on outside tubing surface and the temperature difference between outside tubing and inside casing surface

$h_{r}$ : Heat transfer coefficient for radiation based on the outside tubing surface and the temperature difference between the outside tubing and inside casing surface

$K_{\text {cas }}$ : Thermal conductivity of the casing material at the average casing temperature

$K_{\text {cem }}$ : Thermal conductivity of the cement at the average cement temperature and pressure

$\lambda: \quad$ The friction coefficient, dimensionless

$\gamma_{g}: \quad$ Euler constant 1.781

$\rho: \quad$ Density $\left(\mathrm{kg} / \mathrm{m}^{3}\right)$

$\theta: \quad$ Inclination angle flow conduit.

\section{Conflict of Interests}

The authors declare that there is no conflict of interests regarding the publication of this paper.

\section{Acknowledgments}

This research was supported by the Key Program of NSFC (Grant no. 70831005) and the Key Project of China Petroleum and Chemical Corporation (Grant no. GJ-73-0706).

\section{References}

[1] H. D. Beggs and J. R. Brill, "A study of two-phase flow in inclined pipes," Journal of Petroleum Technology, vol. 25, pp. 607-617, 1973.

[2] H. Mukherjee and J. P. Brill, "Liquid holdup correlations for inclined two-phase flow," Journal of Petroleum Technology, vol. 35, no. 5, pp. 1003-1008, 1983.

[3] H. Mukherjee and J. P. Brill, "Empirical equations to predict flow patterns in two-phase inclined flow," International Journal of Multiphase Flow, vol. 11, no. 3, pp. 299-315, 1985.

[4] E. T. Hurlburt and T. J. Hanratty, "Prediction of the transition from stratified to slug and plug flow for long pipes," International Journal of Multiphase Flow, vol. 28, no. 5, pp. 707-729, 2002.

[5] O. Cazarez-Candia and M. A. Vásquez-Cruz, "Prediction of pressure, temperature, and velocity distribution of two-phase flow in oil wells," Journal of Petroleum Science and Engineering, vol. 46, no. 3, pp. 195-208, 2005.
[6] Y. Taitel, O. Shoham, and J. P. Brill, "Simplified transient solution and simulation of two-phase flow in pipelines," Chemical Engineering Science, vol. 44, no. 6, pp. 1353-1359, 1989.

[7] L.-B. Ouyang and K. Aziz, "Transient gas-liquid two-phase flow in pipes with radial influx or efflux," Journal of Petroleum Science and Engineering, vol. 30, no. 3-4, pp. 167-179, 2001.

[8] J. P. Fontanilla and K. Aziz, "Prediction of bottom-hole conditions for wet steam injection wells," Journal of Canadian Petroleum Technology, vol. 21, no. 2, 8 pages, 1982.

[9] S. M. F. Ali, "A comprehensive wellbore stream/water flow model for steam injection and geothermal applications," Society of Petroleum Engineers Journal, vol. 21, no. 5, pp. 527-534, 1981.

[10] Z. Wu, J. Xu, X. Wang, K. Chen, X. Li, and X. Zhao, "Predicting temperature and pressure in high-temperature-high-pressure gas wells," Petroleum Science and Technology, vol. 29, no. 2, pp. 132-148, 2011.

[11] J. Xu, M. Luo, S. Wang, B. Qi, and Z. Qiao, "Pressure and temperature prediction of transient flow in HTHP injection wells by Lax-Friedrichs method," Petroleum Science and Technology, vol. 31, no. 9, pp. 960-976, 2013.

[12] S. K. Godunov, "A difference method for numerical calculation of discontinuous solutions of the equations of hydrodynamics," Matematicheskii Sbornik, vol. 47, no. 89, pp. 271-306, 1959.

[13] J. Li and G. Chen, "The generalized Riemann problem method for the shallow water equations with bottom topography," International Journal for Numerical Methods in Engineering, vol. 65, no. 6, pp. 834-862, 2006.

[14] D. John and J. R. Anderson, Computational Fluid DynamicsThe Basics with Applications, McGraw-Hill, New York, NY, USA, 1995.

[15] X.-W. Liao and J.-L. Feng, "Pressure-temperature coupling calculation of transient wellbore heat transfer in deep geopressured gas reservoir," Petroleum Exploration and Development, vol. 32, no. 1, pp. 67-69, 2005.

[16] E. F. Toro, Riemann Solvers and Numerical Methods for Fluid Dynamics: A Practical Introduction, Springer, Berlin, Germany, 1997.

[17] J. Xu, J. Hu, M. Luo, S. Wang, B. Qi, and Z. Qiao, "Optimisation of perforation distribution in HTHP vertical wells," Canadian Journal of Chemical Engineering, vol. 91, pp. 332-343, 2011. 


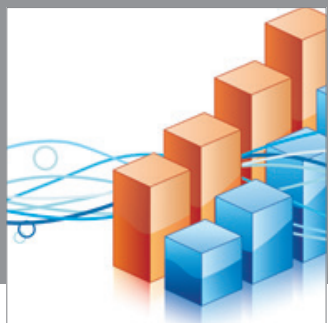

Advances in

Operations Research

mansans

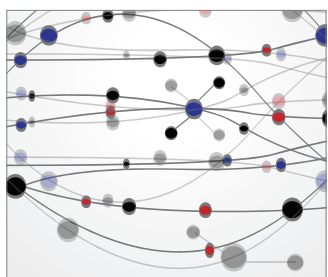

The Scientific World Journal
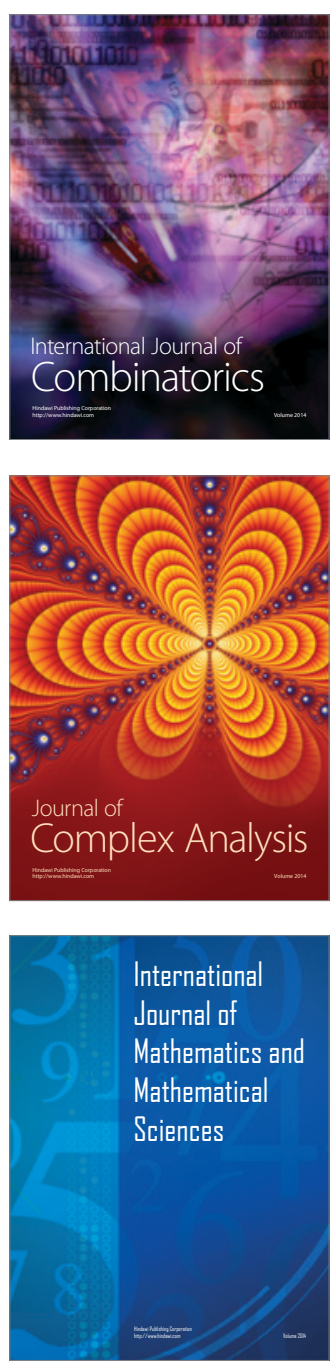
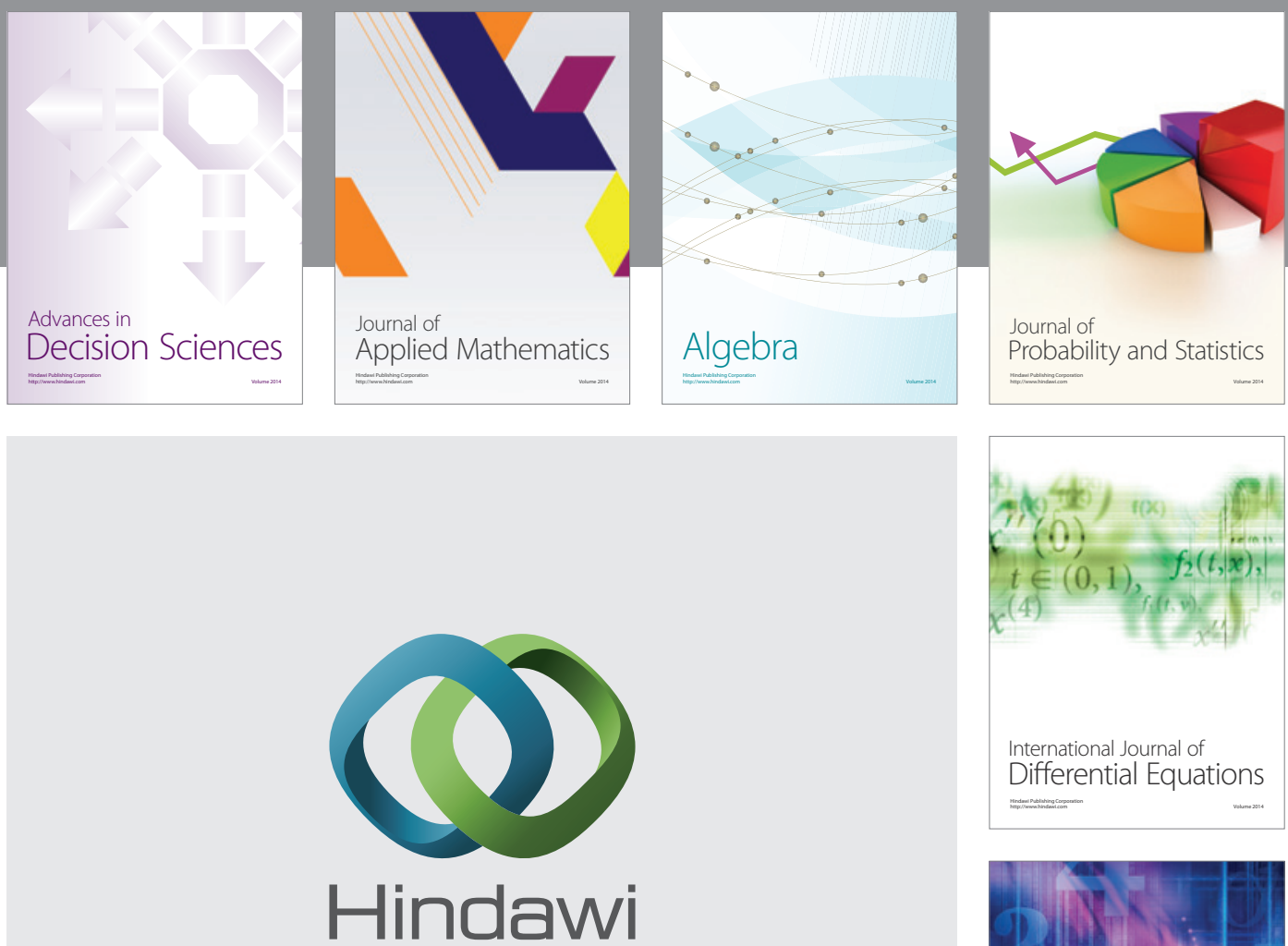

Submit your manuscripts at http://www.hindawi.com
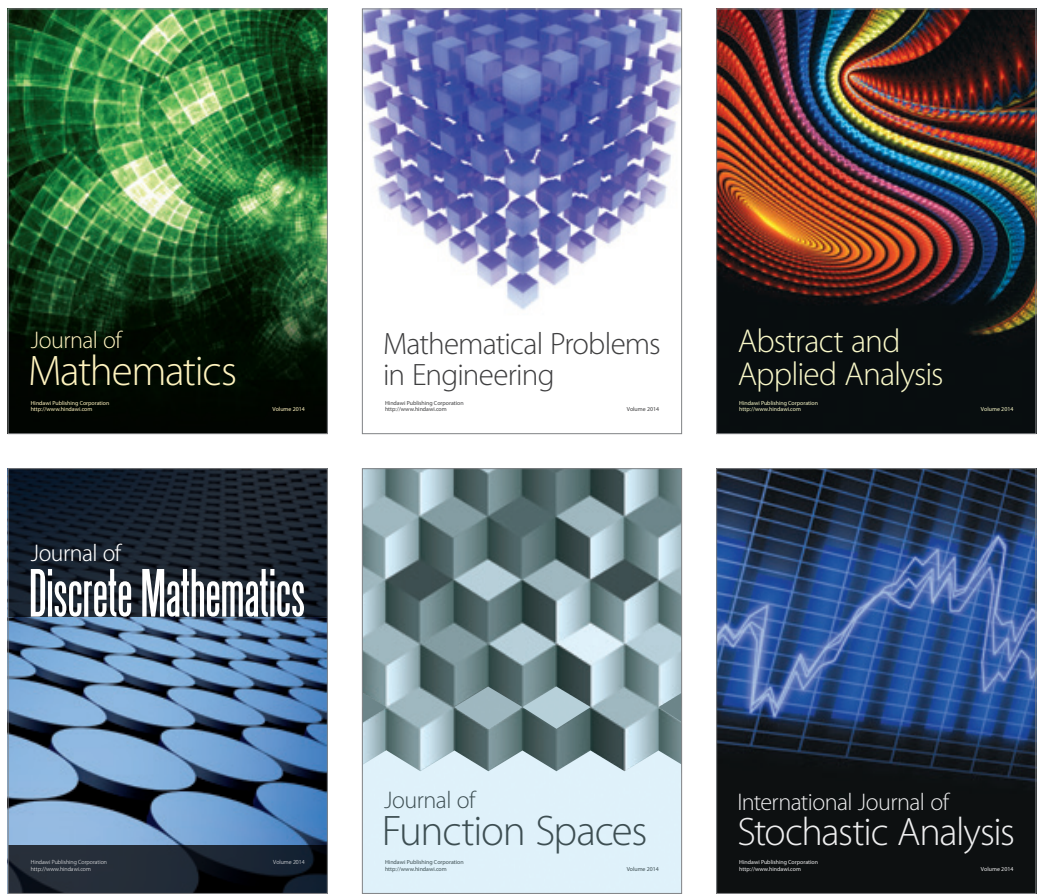

Journal of

Function Spaces

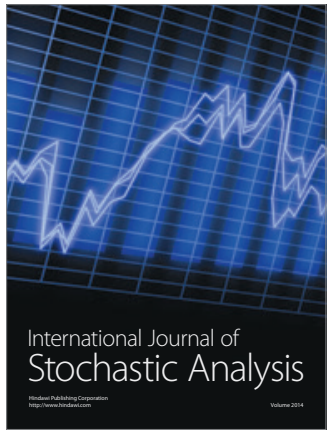

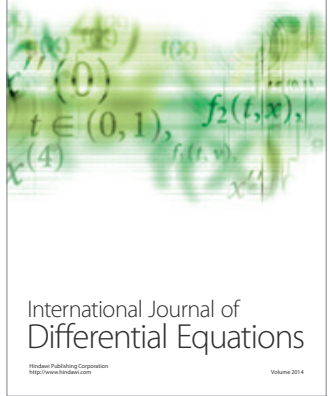
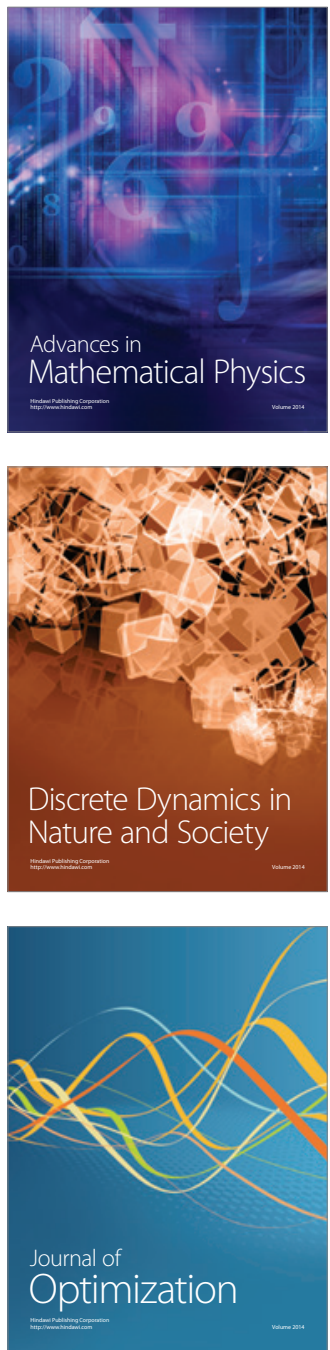